\title{
A RADISH (RAPHANUS SATIVUS L.) E3 UBIQUITIN LIGASE GENE $R H A 2 b$ ENHANCES SEED DORMANCY AND TOLERANCE TO PREHARVEST SPROUTING IN TRANSGENIC WHEAT (TRITICUM AESTIVUM L.)
}

\author{
LI, D. B." - LYU, G. Z.\# - JIANG, Y. M. - NIU, H. B. - WANG, X. - YIN, J. \\ National Engineering Research Center for Wheat, State Key Laboratory of Wheat and Maize \\ Crop Science, Collaborative Innovation Center of Henan Grain Crop, Henan Agricultural \\ University, Zhengzhou 450002, China \\ *Corresponding author \\ e-mail:xmzxyj@126.com; phone: +86-371-6355-8203 \\ ${ }^{\#}$ These authors contributed equally to this work \\ (Received $9^{\text {th }}$ Jun 2019; accepted $28^{\text {th }}$ Aug 2019)
}

\begin{abstract}
The RHA2b gene from radish encodes a transcription factor that is involved in abscisic acid (ABA) signal transduction and is associated with seed dormancy and preharvest sprouting. RsRHA2b gene may have the function to improve dormancy and tolerance to PHS in transgenic wheat. The $R s R H A 2 b$ gene was cloned and transferred into Zhengmai 9023 via Agrobacterium-mediated stem apex transformation. The results of RT-PCR and southern blot indicated that the RsRHA2b gene was integrated into the genomes of the wheat transformants. Seed dormancy and PHS tolerance was significantly enhanced in transgenic plants that stably expressed RsRHA2b. In addition, in the presence of $0.5 \mu \mathrm{mol} / \mathrm{L}$ $\mathrm{ABA}$ and $5 \mu \mathrm{mol} / \mathrm{L} \mathrm{ABA}$, a significant reduction in weight was found between the transgenic wheat plants and the non-transgenic Zhengmai 9023 plants. Furthermore, the expression of genes involved in RHA2b-mediated ABA signal transduction, such as $A B I 5, F U S 3$ and $M A L$, significantly increased at the second leaf stage in ABA-treated transgenic wheat plants. These results show that RsRHA2b improves dormancy and preharvest sprouting tolerance in transgenic wheat.
\end{abstract}

Keywords: agrobacterium-mediated stem apex transformation, southern blot, abscisic acid, signal transduction, reverse transcription-polymerase chain reaction

\section{Introduction}

Preharvest sprouting (PHS) in wheat (Triticum aestivum L.) is the germination of grains in the ears when long-range rainfall or damp conditions occur prior to harvest (Li et al., 2004). PHS is an important issue, which is is not conducive to the stability of wheat production worldwide (Xiao et al., 2002). Seed viability and the hydrolysis of starch and protein in the endosperm are always reduced after PHS (Mohan, 2008). Moreover, PHS creates a favorable environment for serious infections by saprophyte fungi and thus causes heavy losses to grain yield (Castor and Frederiken, 1977). Therefore, there is a need to breed for increased resistance to PHS. Molecular breeding may be an effective method to reduce PHS susceptibility. Wheat is an important cereal crop worldwide, and it is a staple food for many populations. Genetically modified (GM) crops can act as powerful complement to the crops produced by laborious and time consuming conventional breeding methods to meet the worldwide demand for quality foods and help fight malnutrition by enhanced yield, nutritional quality and increased resistance to various biotic and abiotic stresses (Kamthan et al., 2016). PHS and dormancy in wheat have been focuses of numerous researches. TaRHA2b and $R s R H A 2 b$ gene may play an important role in seed 
dormancy during germination, which could be used to improve the PHS resistance of wheat. Because of homologous co-suppression in transgenic plants, exogenous genes are usually used for genetic manipulation.

To date, most researchers have agreed that PHS is the result of interactions between genotypes and the environment. Seed dormancy is an adaptive trait that inhibits seed germination and is positively correlated with resistance to PHS (Derera et al., 1977; Gerjets et al., 2010; Gubler et al., 2005; Chen et al., 2008b). The resistance of a seed to PHS is partially influenced by the external environment, but seed dormancy is the most critical determinant (Kucera et al., 2005; Holdsworth et al., 2008). As has been confirmed in many species, seed dormancy is influenced by the internal abscisic acid (ABA) content and the sensitivity to ABA (Groot and Karssen, 1992; Tan et al., 1997). ABA plays a critical role in plant development. Therefore, ABA, including its metabolism and signaling, has long been a focus of research (Grill and Himmelbach, 1998; Raghavendra et al., 2010; Zhu, 2002). ABA is important for plant growth and developmental processes, such as seed maturation, germination and seedling growth. $\mathrm{ABA}$ also regulates plant adaptation to various environmental challenges, including drought, salt, cold and other abiotic stresses (Nambara and Marion-Poll, 2003; Yamaguchi Shinozaki and Shinozaki, 2006; Lopez-Molina et al., 2001). During seed maturation, the $\mathrm{ABA}$ content in the seed increases significantly, resulting in strong dormancy. After the mature seed absorbs water, it quickly changes from a state of dormancy to a state of germination.

Many regulatory factors and response regulators in ABA signaling act in the signal transduction cascade during seed maturation and in young seedlings. These factors include $\mathrm{ABA}$ receptors, regulators and really interesting new gene (RING) finger transcription factors, which regulate the regulatory factors via ubiquitination and via the expression of ABA downstream response regulators (Koornneef et al., 1984). The genes related to ABA include ABI5, FUS3, MAL, and so on (Chiu et al., 2012; Huang et al., 2012; Li et al., 2011). The ubiquitin-proteasome pathway comprises two major components, the ubiquitination machinery, which attaches a ubiquitin or a polyubiquitin chain to a substrate, and the $26 \mathrm{~S}$ proteasome, which degrades poly-ubiquitintagged protein substrates (Dreher and Callis, 2007; Moon et al., 2004; Schwechheimer and Schwager, 2004; Smalle and Vierstra, 2004). The ubiquitination of substrate proteins requires three types of enzymes, including E1 enzymes, which are ubiquitinactivating enzymes; E2 enzymes, which are ubiquitin-conjugating enzymes; and E3 enzymes, which are ubiquitin ligases. These three enzyme types act sequentially to catalyze the covalent attachment of the 76-amino-acid ubiquitin to the target protein. The E3 ligases recruit specific substrates and are the key determinants of the specificity of ubiquitination (Smalle and Vierstra, 2004).

RING finger proteins have motifs containing conserved cysteines and histidines. The RING motifs can be classified into three subgroups, including C3HC4 (RING$\mathrm{HC}$ ), $\mathrm{C} 3 \mathrm{H} 2 \mathrm{C} 3$ (RING-H2) and modified RING domain proteins. RING finger proteins always mediate protein-protein interactions and the formation of multi-protein complexes (Borden, 2000; Stone et al., 2005). At least 477 RING motif-containing proteins that play a critical role in plant developmental processes have been found in Arabidopsis (Vierstra, 2009). Some evidence has indicated that RING class E3 enzymes are critical for regulating ABA signaling and are associated with abiotic stress responses in plants (Ko et al., 2006; Zhang et al., 2005, 2007). RHA2a and RHA2b, which are RING finger E3 ligases, can positively regulate the ABA-mediated 
inhibition of germination and act in the early seedling stage. RHA2a can activate positive regulators by mono-ubiquitinating and stabilizing certain key regulators of the ABA signaling pathway (Bu et al., 2009). ANAC019 and ANAC055, two closely related NAC family proteins, were identified as RHA2a-interacting proteins (Bu et al., 2008; Jiang et al., 2009). RHA2b acts additively with RHA2a in regulating ABA signaling. Seeds over-expressing RHA2 $a$ and $R H A 2 b$ showed increased sensitivity to exogenous ABA, increased dormancy and a significant reduction in germination capacity. RHA2b targets MYB30 degradation to regulate ABA signal transduction (Zheng et al., 2018). Although some progress has been made in understanding the molecular and biological functions of $R H A 2 b$ in Arabidopsis, the molecular mechanism of $R H A 2 b$-mediated ABA signaling is largely unknown. The AtRHA $2 b$ gene encodes an active E3 ubiquitin ligase that plays a positive regulatory role in ABA signaling and stress responses. The over-expression of AtRHA2b leads to ABAassociated phenotypes, such as ABA hyper-sensitivity in seed germination and seedling growth (Li et al., 2011).

In our previous studies, TaRHA2b and $R s R H A 2 b$ genes were cloned from wheat and radish (Raphanus sativus L.), respectively. The results showed that the proteins encoded by TaRHA2b and RsRHA2b were consisted of Zinc finger RING-type profiles. Blast and phylogenetic analysis showed that the protein encoded by TaRHA $2 b$ and RsRHA2b shared the identity with AtRHA2b from the Arabidopsis. The expression of TaRHA2b and RsRHA2b genes was significantly tissue-specific. The sensitivity of the TaRHA2b and RsRHA2b genes to ABA was significantly increased (Li et al., 2019a). The differences in nucleotide polymorphism sites of $R s R H A 2 b$ gene resulted in differences in the three cleavage sites of BstD102I, Cfr10I, and HpaII/MspI (Li et al., 2019b). These results facilitate the exploration of the correlation between the TaRHA2b gene and wheat's enhanced resistance to PHS and the development of molecular markers for resistance to PHS.

In the present study, the RsRHA2b gene from radish was cloned and then transferred into wheat using Agrobacterium tumefaciens. Seed dormancy and ABA sensitivity were significantly enhanced in transgenic wheat lines. These results displayed that $R s R H A 2 b$ gene has the function to improve dormancy and tolerance to PHS in transgenic wheat.

\section{Materials and methods}

To clone the RsRHA2b gene, mRNA was isolated from the embryos of a local radish variety, "New generation 791". The elite Chinese bread wheat cultivar Zhengmai 9023, a hexaploid wheat cultivar with weak resistance to PHS, which is widely cultivated in Henan Province, was used for transformation.

\section{Construction of a binary vector}

The coding sequence and 3'-untranslated region (3'-UTR) of the RsRHA2b gene were amplified from radish cDNA via PCR with primers $R s R H A 2 b$-F2/RsRHA2b-R2 (Table 1). The PCR-amplified products were cloned into TA vectors (Takara, Dalian, China) and sequenced. A binary vector was constructed based on pCAMBIA3301. The TA vectors including the RsRHA2b gene and the pCAMBIA3301 vector were digested with $B g l I I$ and BstEII. The two digested fragments were ligated together using T4 ligase. The resulting binary vector carried the phosphinothricin gene (bar) for selection. 


\section{Agrobacterium-mediated stem apex transformation of wheat}

Wheat seeds were sterilized with $0.1 \% \mathrm{HgCl}_{2}$ for $10 \mathrm{~min}$, then washed five times with sterilized distilled water. The sterilized seeds were germinated at $25{ }^{\circ} \mathrm{C}$ in the dark for 2-3 days on filter-containing plates. When the coleoptiles of the seeds reached a certain height $(2-4 \mathrm{~cm})$, the shoots were cut using a clean knife. Then, the stem apexes were exposed. Finally, 2-4 $\mu \mathrm{L}$ of resuspended Agrobacterium harboring the RsRHA $2 b$ gene and that had been induced with $100 \mu \mathrm{mol} / \mathrm{L}$ acetosyringone was dropped onto the stem apex for inoculation.

The inoculated seedlings were maintained on medium-containing plates until new leaves grew out, and then the seedlings were transferred into pots. Selection for resistance was performed by spraying the seedlings with a solution containing $160 \mathrm{mg} / \mathrm{L}$ basta when the seedlings had 3-4 leaves. The resistant plants were planted in the field.

\section{Propagation of the transgenic lines}

The seeds were harvested from the field in 2011. Then, they were grown in a digital biochemical incubator after rigorous screening. When the seedlings had 3-4 leaves, selection for resistance was performed by spraying the seedlings with a solution containing $160 \mathrm{mg} / \mathrm{L}$ basta. Genomic DNA and RNA were extracted from young wheat leaves. Then, PCR, reverse transcription-polymerase chain reaction (RT-PCR) and quantitative real-time polymerase chain reaction (qRT-PCR) analyses were conducted on the resistant plants. The seeds of RsRHA2b transgenic plants were harvested while the plants were in the digital biochemical incubator. Then, they were grown in the field. They were then grown and screened as in prior years.

\section{DNA and RNA extraction}

Genomic DNA was extracted from young wheat leaves. Total RNA was isolated from seeds and leaves using TRIzon reagent (CoWin Biotech Co., China) according to the manufacturer's instructions and treated with RNase-free DNase I (Tiangen, China) before final precipitation with ethanol.

\section{PCR, RT-PCR analysis and southern blot}

For the PCR analysis of transgenic plants, a 565 bp $R s R H A 2 b$ product was amplified using PCR with the primers RsRHA2b-F1/RsRHA2b-R1 (Table 1), which were designed based on pCAMBIA3301-RsRHA2b. The primers were specific for radish.

For RT-PCR analyses of transgenic plants, approximately $5 \mu \mathrm{g}$ of total RNA from the shoots of transgenic plants was reverse-transcribed into cDNA using reverse transcriptase (Tiangen, China) and oligo-dT ${ }_{18}$ primers. PCR amplifications were performed using the primers RsRHA2b-F2/RsRHA2b-R2.

Southern blot analysis was performed using standard procedures. Genomic DNA samples $(30 \mu \mathrm{g})$ from PCR-positive transgenic wheat plants were digested with HindIII overnight at $37{ }^{\circ} \mathrm{C}$. The DNA fragments were separated on $1.0 \%(\mathrm{w} / \mathrm{v})$ agarose gels and transferred to Hybond $\mathrm{N}^{+}$nylon membranes. The PCR-amplified fragment to be used as a probe was further purified and labeled with digoxigenin (DIG)-dUTP using a DIGHigh Prime Kit (Roche, Manheim, Germany). Hybridization was conducted at $68{ }^{\circ} \mathrm{C}$. Hybridization and detection were performed using a DIG Nucleic Acid Detection Kit (Roche) according to the manufacturer's instructions. 


\section{qRT-PCR analysis of transgenic wheat plants}

For qRT-PCR, the RNA from wheat leaves at $12 \mathrm{~h}, 24 \mathrm{~h}, 48 \mathrm{~h}$ after treatment with 0 $\mu \mathrm{mol} / \mathrm{L}, 0.5 \mu \mathrm{mol} / \mathrm{L}$, or $5 \mu \mathrm{mol} / \mathrm{L}$ ABA was used for reverse transcription (Toyobo, Osaka, Japan). qRT-PCR was performed in a volume of $20 \mu \mathrm{L}$ containing SYBR Green I PCR Master Mix (Promega, Madison, USA) 10 pmol each of forward and reverse gene-specific primers (Table 1), and cDNA $(0.2 \mu \mathrm{mol} / \mathrm{L})$. Gene-specific primers were designed using Primer Premier 5 software. The wheat ACTIN gene was co-amplified as an internal control to normalize the total amount of cDNA present in each reaction and to eliminate the differences among the samples. PCR amplification was performed in an iQ5 Cycler (Bio-Rad) under the following conditions: $95^{\circ} \mathrm{C}$ for $3 \mathrm{~min}$, followed by 39 cycles of $94{ }^{\circ} \mathrm{C}$ for $10 \mathrm{~s}, 60{ }^{\circ} \mathrm{C}$ for $20 \mathrm{~s}$, and $72{ }^{\circ} \mathrm{C}$ for $30 \mathrm{~s}$. The plate was read at $72{ }^{\circ} \mathrm{C}$ for $30 \mathrm{~s}$. To determine the specificity of each PCR primer pair, a melt curve was generated by incubating the reaction at $95{ }^{\circ} \mathrm{C}$ for $10 \mathrm{~s}$, cooling to $65{ }^{\circ} \mathrm{C}$ for $10 \mathrm{~s}$ and increasing to $95^{\circ} \mathrm{C}$ at a rate of $0.5^{\circ} \mathrm{C} / 10 \mathrm{~s}$.

Table 1. Primer sequences used for PCR, RT-PCR, qRT-PCR and southern blot analysis

\begin{tabular}{c|c}
\hline Name & Sequence (5'-3') \\
\hline$R s R H A 2 b-\mathrm{F} 1$ & CCATGGTCCAGCAACAGCAGCAAACA \\
$R s R H A 2 b-\mathrm{R} 1$ & GGTGACCGGAGAAAGCCGCGAGATTAAA \\
qRT- $R s R H A 2 b-\mathrm{F}$ & TGCCCGAGGAGGTCAAGGAG \\
qRT- $R s R H A 2 b-\mathrm{R}$ & CGTGATTGGATGGCTACTATACAAAGTG \\
$R s R H A 2 b-\mathrm{F} 2$ & AGATCTATGGGGTTACAAGGTCAACTCT \\
$R s R H A 2 b-\mathrm{R} 2$ & GGTGACCGGAGAAAGCCGCGAGATTAAA \\
Trans(gDNA)-F & AGATCTATGGGGTTACAAGGTCAACTCT \\
Trans(gDNA)-R & GGTGACCGGAGAAAGCCGCGAGATTAAA \\
Trans(cDNA)-F & AGATCTATGGGGTTACAAGGTCAACTCT \\
Trans(cDNA)-R & GGTGACCGGAGAAAGCCGCGAGATTAAA \\
Southernblot(probe)-F & AGATCTATGGGGTTACAAGGTCAACTCT \\
Southernblot(probe)-R & GGTGACCGGAGAAAGCCGCGAGATTAAA \\
TaACTIN-F & GCTGTTCCAGCCATCTCATGT \\
TaACTIN-R & CGATCAGCAATTCCAGGAAAC \\
$A B I 5-\mathrm{F}$ & GACGGAAGAAGCGGCAGTG \\
$A B I 5-\mathrm{R}$ & TCTGAAGCAGCACGAGAAAGG \\
$F U S 3-\mathrm{F}$ & GTGGCTGGGTTGCGAGTTAT \\
$F U S 3-\mathrm{R}$ & ATCCTGCTCTTGTTGTTTGGC \\
$M A L-\mathrm{F}$ & CTTCCTGTGCTGCCTATTCTG \\
$M A L-\mathrm{R}$ & CCACATGCACTGGACGATAAC \\
\hline
\end{tabular}

\section{Seed germination test}

Intact spikes were harvested at physiological maturity. Grains from the wheat spikes were hand-threshed. The dormant seeds were used for further analysis. Thirty sound grains of each plant were placed crease down on moist filter paper in Petri dishes at $20{ }^{\circ} \mathrm{C}$ in the dark for $7 \mathrm{~d}$. The germinated grains were counted every day and 
calculated in terms of the germination index $(\mathrm{GI}) . \mathrm{GI}=(7 \mathrm{n} 1+6 \mathrm{n} 2+\ldots+1 \mathrm{n} 7) / 7 \mathrm{~N}$, where $\mathrm{n} 1, \mathrm{n} 2, \mathrm{n} 3, \ldots \mathrm{n} 7$ are the number of grains that had germinated on the 1 st day, 2nd day, ...7th day, and $\mathrm{N}$ is the total number of grains in the assay. The grains from each plant composed a replication, and the experiments were performed in three replicates. Additionally, 30 sound grains of each plant were placed crease down on moist filter paper in Petri dishes at $20{ }^{\circ} \mathrm{C}$ in normal conditions $\left(16 \mathrm{~h}\right.$, light: $8 \mathrm{~h}$, dark; $\left.20{ }^{\circ} \mathrm{C}\right)$. The germination capacity on the $39^{\text {th }}$ day after sowing was recorded.

\section{Spike sprouting test}

Ten spikes of each T5-generation transgenic line harvested from the experimental field were inserted into floral foam (floral foam can retain water to prevent the detached wheat spikes from wilting) at approximately $20-25{ }^{\circ} \mathrm{C}$ in a greenhouse. Misting was controlled by a humidifier and checked automatically for $4 \mathrm{~d}$. Under these conditions, a high relative humidity (>98\%) was maintained.

\section{$A B A$ sensitivity assay}

Wheat seeds from the transgenic lines and from the non-transgenic Zhengmai 9023 whose dormancy had been broken were tested for ABA sensitivity. Seeds were germinated at $20{ }^{\circ} \mathrm{C}$. At the second leaf stage, they were treated with different concentrations of ABA, including $0 \mu \mathrm{mol} / \mathrm{L}, 0.5 \mu \mathrm{mol} / \mathrm{L}$ and $5 \mu \mathrm{mol} / \mathrm{L}$ ABA. Fourteen seedlings from each transgenic line and from non-transgenic Zhengmai 9023 were assayed after being treated for13 d (Saad et al., 2013). The initial fresh weight and final fresh weight of the plants were measured. Then, the difference in weight was calculated. The experiments were performed on three replicates.

\section{Statistical analysis}

The GIs, germination capacity and average weight difference of each line were analyzed using Excel software, and Student's $t$ test was calculated for relevant data using SPSS software. The figures were created using Origin8 software and Adobe Photoshop CS5 software.

\section{Results}

\section{Cloning and analysis of the RsRHA2b gene}

The EST database was BLASTed based on the amino acid sequence encoded by the Arabidopsis RING finger gene AtRHA2b (Gene ID: 814644). Many highly homologous ESTs were found. A cDNA sequence including the entire ORF of the radish RING finger gene $R H A 2 b$ was found using artificial splicing and software analysis based on the radish database. The length of the entire sequence was $697 \mathrm{bp}$ (Fig. 1). The ID of the RsRHA2b gene was KR349970. The entire coding sequence, plus a 93 bp sequence in the 3'-UTR, was amplified from radish cDNA and was 565 $\mathrm{bp}$ in length. The sequence was then used to construct the binary vector pCAMBIA3301-RsRHA2b (Fig. 2). The constructed vector was further confirmed by sequence analysis (Fig. 1) and was then transferred into Agrobacterium tumefaciens EHA105. Transgenic wheat plants were obtained to investigate the function of RsRHA2b. 


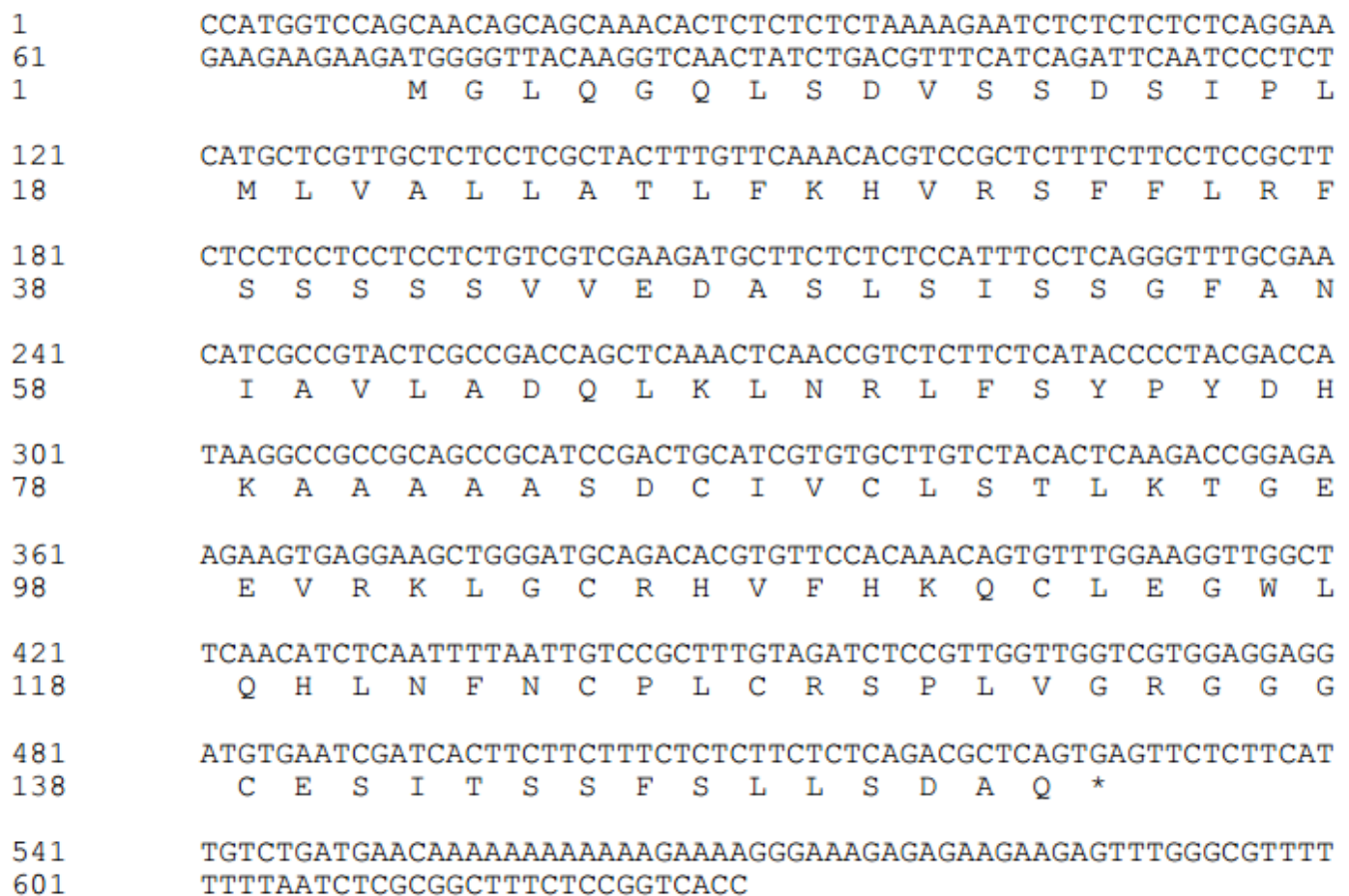

Figure 1. Nucleotide and deduced amino acid sequences of the RsRHA2b gene

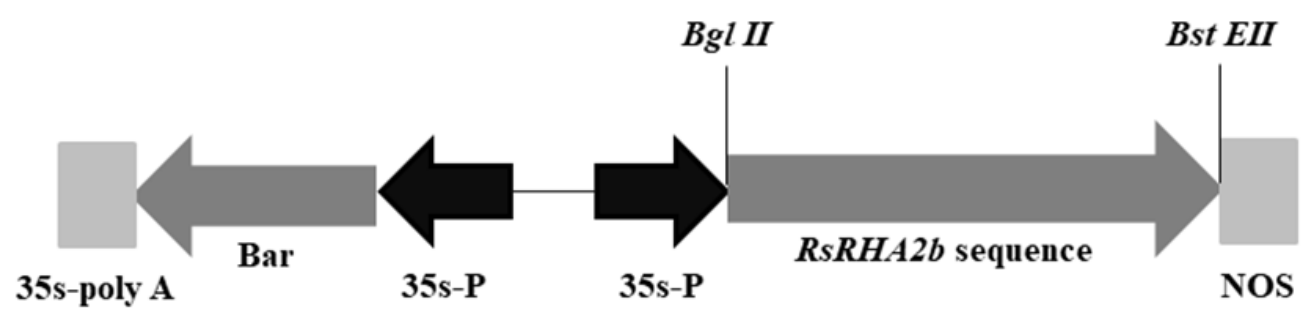

Figure 2. Structure of the binary vector pCAMBIA3301-RsRHA2b, which was used for transformation. RsRHA2b sequence, RsRHA2b coding sequence plus 93 bp sequence at 3'UTR; 35S-P, 35S-promoter; Bar, phosphinothricin gene

\section{Identification of transgenic wheat plants}

The transgenic plants were identified using PCR and RT-PCR. As shown in Figure 3, the RsRHA2b gene fragment was found in the genome of transgenic wheat, and $R s R H A 2 b$ transcripts were also detected. These results showed that the $R s R H A 2 b$ gene was stable in the wheat genome and was stably expressed. Each of the five lines (L1, L2, L3, L4 and L5) showed a single band, indicating independent transformation events (Fig. 4). As expected, there was no significant hybridization signal from WT DNA. These results indicated that the RsRHA2b gene was integrated into the genomes of the wheat transformants in the selected lines and was inherited by the progeny as a single copy. However, the segregation was not consistent with heredity of a single-copy gene. Ultimately, four transgenic lines, 1471 (100 wheat heads), 1477 (102 wheat heads), 1492 (105 wheat heads) and 1569 (103 wheat heads), were selected for further characterization. Their morphology was similar to that of the non-transgenic Zhengmai 9023. 


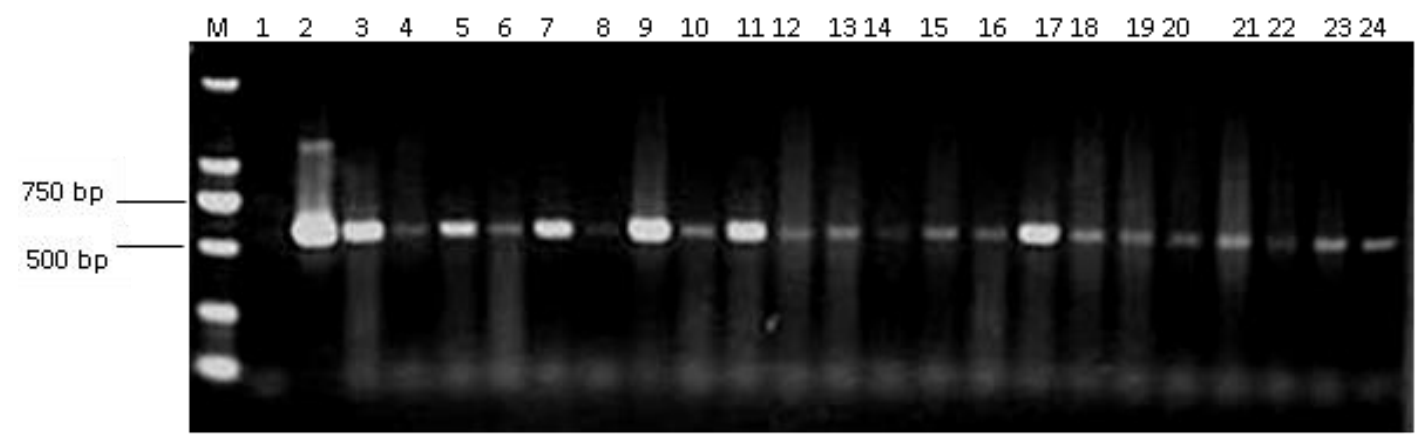

Figure 3. Identification of transgenic plants by PCR and RT-PCR analyses. DNA was extracted from the leaves of each T5 transgenic line and from non-transgenic Zheng9023. RNA was extracted from the shoots of each T5 transgenic line and non-transgenic Zheng9023. (3-13) DNA fragments between the RsRHA2b coding sequence and the NOS sequence amplified by primers $R s R H A 2 b-F / R s R H A 2 b-R$. (14-24) $R T-P C R$ products of the RsRHA2b sequence amplified from cDNA using primers. RsRHA2b-F/RsRHA2b-R. M, marker 2000; 1, nontransgenic Zheng9023; 2, plasmid pCAMBIA3301-RsRHA2b; 3-13, transgenic lines (DNA); 1424 , transgenic lines (cDNA)

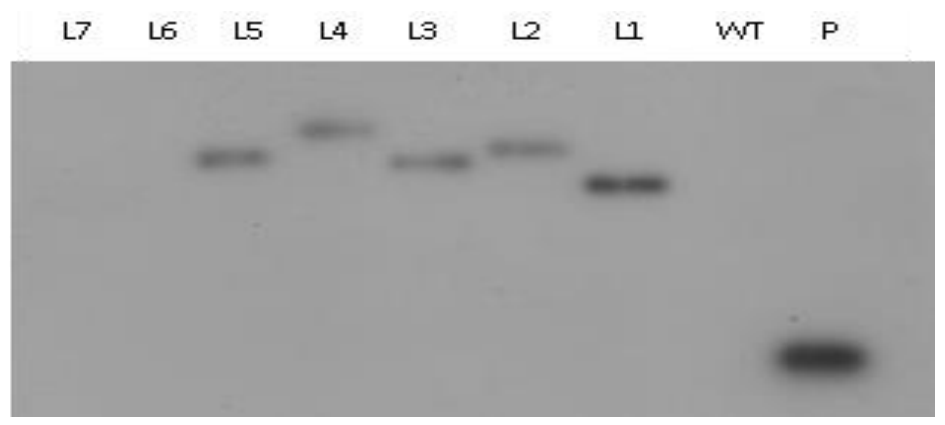

Figure 4. Southern blot analysis. L1-7, seven randomly selected T2 PCR-positive lines (L1-7); WT, non-transgenic Zheng9023; P: purified probe product (positive control). Thirty micrograms of HindIII-digested total wheat leaf DNA was fractionated on a 1\% agarose gel, blotted onto a nylon membrane, and hybridized with a probe labeled with DIG-11-dUTP

\section{RsRHA2b has an important role in the regulation of RsRHA2b-mediated seed dormancy}

To assay the seed dormancy of transgenic wheat, GIs were investigated using the seeds from the T5 generation. The GIs were significantly lower in the transgenic wheat lines than in the non-transgenic Zhengmai 9023 (Table 2). The GIs of the seeds of the transgenic lines ranged from 0.098 to 0.387 , a significant reduction, up to $89.9 \%$. In particular, the GI of transgenic line 1477 was 0.098 . The germination capacity of the transgenic lines ranged from 0.367 to 0.724 , a significant reduction of up to $63.3 \%$. In particular, the germination capacity of the transgenic line 1477 was 0.367 . GI value (less than 0.4) was defined as resistance to spike germination. GI value (0.4-0.8) is medium resistance. GI value (more than 0.8 ) is prone to spike germination (Ma et al., 2014). The GI values of the seeds of the four transgenic lines in this study were all less than 0.4 , while the average GI values of the control group were 0.973 . Therefore, the retarded seed germination in the transgenic wheat with $R S R H A 2 b$ gene is stably inherited, and RsRHA2b can increase seed dormancy. 
Table 2. Seed germination indexes and germination capacity (on the $39^{\text {th }}$ day after germination) in different T5 transgenic wheat compared with those of non-transgenic Zhengmai 9023

\begin{tabular}{c|c|c}
\hline Transgenic line & Germination index $^{\mathbf{a}}(\mathbf{G I})$ & Germination capacity $^{\mathbf{b}}$ \\
\hline 1471 & $0.217 \pm 0.010 \mathrm{~b}$ & $0.650 \pm 0.024 \mathrm{~b}$ \\
1477 & $0.098 \pm 0.003 \mathrm{~b}$ & $0.367 \pm 0.047 \mathrm{~b}$ \\
1492 & $0.244 \pm 0.017 \mathrm{~b}$ & $0.600 \pm 0.024 \mathrm{~b}$ \\
1569 & $0.387 \pm 0.007 \mathrm{~b}$ & $0.724 \pm 0.024 \mathrm{~b}$ \\
CK $^{\mathrm{c}}$ & $0.973 \pm 0.012 \mathrm{a}$ & $1.000 \pm 0.000 \mathrm{a}$ \\
\hline
\end{tabular}

${ }^{\mathrm{a}}$ Germination indexes are the average \pm standard error from 10 plants per line

${ }^{\mathrm{b}}$ Germination capacity is the average \pm standard error from 10 plants per line on the $39^{\text {th }}$ day after germination

${ }^{\mathrm{c}} \mathrm{CK}$ : non-transgenic Zhengmai 9023

The letters 'a' and 'b': Significant at $\mathrm{P}<0.05$ with Student's $t$ test

\section{RsRHA2b effectively regulates RsRHA2b-mediated tolerance to PHS in spikes}

To ascertain whether the RsRHA2b gene mediates the PHS response, 10 T5generation plants from each of four transgenic lines, as well as non-transgenic Zhengmai 9023, were assayed for their PHS tolerance. The GI of the transgenic wheat line 1477 was significantly lower than that of the non-transgenic Zhengmai 9023 (Table 2; Fig. 5). Seeds from 1477 had just started to germinate while Zhengmai 9023 had a long first leaf. Spikes of 1477 maintained dormancy while Zhengmai 9023 displayed substantial leaves after $4 \mathrm{~d}$ of misting. The results showed that the RsRHA2b gene can improve PHS tolerance in transgenic wheat plants.

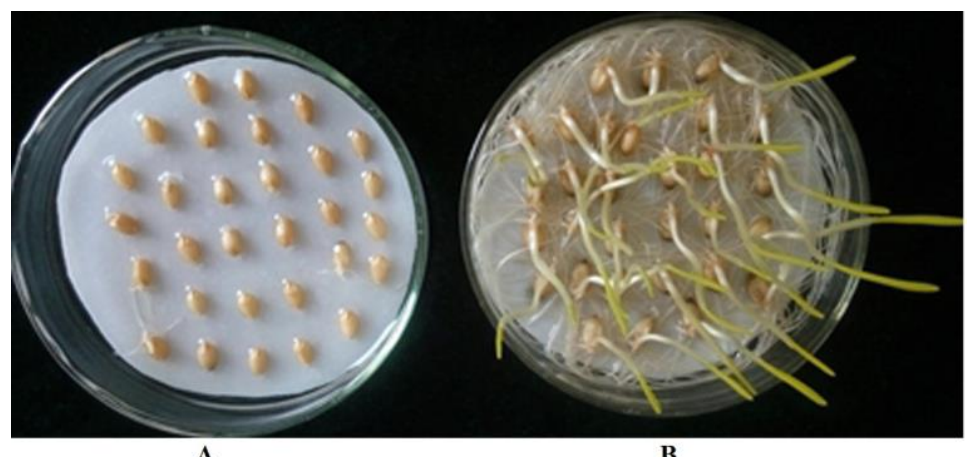

B

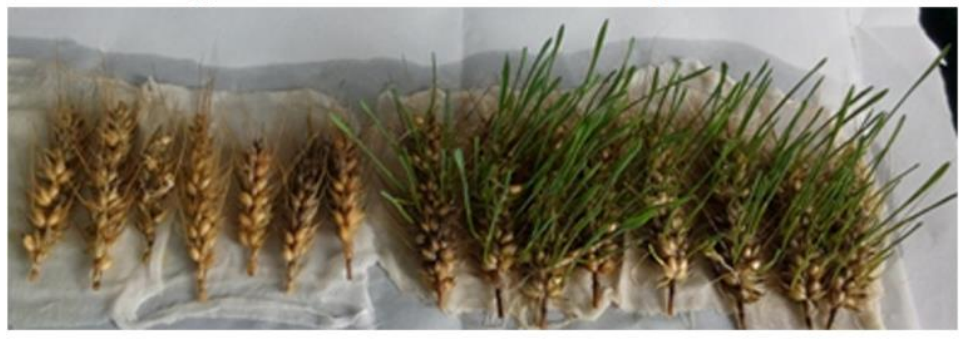

C

D

Figure 5. Seed germination phenotypes of representative seeds and spikes from the T5 transgenic line 1477 and the non-transgenic Zheng9023. (A) Seeds of 1477 and (B) Zheng9023 incubated on a moist filter for $7 d$. (C) Spikes of 1477 and (D) Zheng9023 incubated for $4 d$ under misting conditions 


\section{Higher sensitivity to exogenous $A B A$ in transgenic plants}

The ABA sensitivity of the RsRHA2b transgenic wheat plants was assayed. At the second leaf stage, the plants were transferred to Hoagland's liquid medium containing different concentrations of ABA $(0 \mu \mathrm{mol} / \mathrm{L}, 0.5 \mu \mathrm{mol} / \mathrm{L}$ or $5 \mu \mathrm{mol} / \mathrm{L})$. In the absence of ABA, the weights of transgenic wheat plants and non-transgenic Zhengmai 9023 were $0.701 \mathrm{~g}$ and $0.743 \mathrm{~g}$, respectively (Table 3); there was no significant difference between them. However, in the presence of $0.5 \mu \mathrm{mol} / \mathrm{L} \mathrm{ABA}$, the weights of the transgenic wheat plants and non-transgenic Zhengmai 9023 were $0.417 \mathrm{~g}$ and $0.597 \mathrm{~g}$, respectively (Table 3), and the difference was significant. The inhibitory effect was more apparent when $5 \mu \mathrm{mol} / \mathrm{L}$ ABA was applied. As shown in Table 3, with $5 \mu \mathrm{mol} / \mathrm{L}$ ABA, the weights of transgenic wheat plants and of non-transgenic Zhengmai 9023 were $0.274 \mathrm{~g}$ and $0.448 \mathrm{~g}$, respectively. Taken together, the ABA sensitivity was significantly improved in transgenic wheat lines containing the $R s R H A 2 b$ gene.

Table 3. Weight differences in wheat plants with ABA treatment

\begin{tabular}{c|c|c|c}
\hline Transgenic line & & ABA & \\
\hline & $0 \mu \mathrm{mol} / \mathrm{L}$ & $0.5 \mu \mathrm{mol} / \mathrm{L}$ & $5 \mu \mathrm{mol} / \mathrm{L}$ \\
$\mathrm{CK}^{\mathrm{a}}$ & $0.743 \pm 0.015(\mathrm{~g})$ & $0.597 \pm 0.037(\mathrm{~g}) \mathrm{a}$ & $0.448 \pm 0.006(\mathrm{~g}) \mathrm{a}$ \\
1477 & $0.701 \pm 0.008(\mathrm{~g})$ & $0.417 \pm 0.009(\mathrm{~g}) \mathrm{b}$ & $0.274 \pm 0.015(\mathrm{~g}) \mathrm{b}$ \\
\hline
\end{tabular}

${ }^{\mathrm{a}} \mathrm{CK}$ : non-transgenic Zhengmai 9023

The letters ' $\mathrm{a}$ ' and ' $\mathrm{b}$ ': significant at $\mathrm{P}<0.05$ with Student's $t$ test

\section{RsRHA2b affects the expression of genes associated with $A B A$ signal transduction}

To investigate whether the expression of the RsRHA2b gene affected the ABA signal transduction pathways, the expression levels of several genes associated with ABA were analyzed in the T5 transgenic plants and non-transgenic Zhengmai 9023. Three genes, ABI5 (accession no. AB362818.1), FUS3 (accession no. AM418838.1, homologous cloning) and $M A L$ (accession no. AK334873.1), were found to be closely associated with RsRHA2b. As shown in Figure 6A-C, the expression of three genes showed an obvious steady increase in transgenic wheat plants after ABA treatment. In the presence of $0.5 \mu \mathrm{mol} / \mathrm{L}$ ABA, the expression levels of $A B I 5, F U S 3$, and $M A L$ increased 2.6-fold (48 h, highest level), 25.7-fold ( $24 \mathrm{~h}$, highest level) and 7.2-fold (48 $\mathrm{h}$, highest level), respectively, in the transgenic wheat plants relative to non-transgenic Zhengmai 9023; in the presence of $5 \mu \mathrm{mol} / \mathrm{L} \mathrm{ABA}$, the expression of the three genes increased 3.8-fold (48 h, highest level), 6.8-fold ( $24 \mathrm{~h}$, highest level) and 43.5-fold ( $24 \mathrm{~h}$, highest level), respectively, in the transgenic wheat plants. Taken together, these results indicate that the $R s R H A 2 b$ gene up-regulated the expression of several genes related to seed germination in transgenic wheat.

\section{Discussion}

To solve the problem of PHS in wheat, scholars have conducted much research to explore the mechanism of PHS resistance. The conclusions are as follows. First, studies have focused on the apparent physical and physiological characteristics of wheat seeds, such as spike morphology (Zanetti et al., 2000), the color of the seed coat (Torada and Amano, 2002; Bassoi and Flintham, 2005), seed structure and water absorption (King 
and Von Wettstein-Knowles, 2000), seed dormancy (Andreoli et al., 2006; Hughes et al., 2010), ABA content (Gerjets et al., 2010) and $\alpha$-amylase activity (Major et al., 2001). In the breeding process, grain color, spikelet density, 1000-grain weight and grain width can be used as important reference indexes for wheat varieties resistant to spike germination (Ma et al., 2016). Second, research has focused on the characteristics of genes related to PHS or seed dormancy and the positional cloning of QTLs (Anderson et al., 1993; Chen et al., 2008a; Somers et al., 2007). Third, research has focused on the screening of molecular markers, the mapping of resistance genes and the generation of transgenic lines using genetic engineering methods. PHS resistance in wheat is a quantitative trait, and relevant quantitative trait locuses linked to PHS have been reported on almost all chromosomes (Munkvold et al., 2009; Ogbonnaya et al., 2008). However, PHS-resistant wheat germplasm resources are scarce. Therefore, the use of genetic engineering provides good choices for the improvement of PHS resistance in wheat. The VPI and Trx genes have been used to improve the PHS resistance of wheat (Li et al., 2009; Huang et al., 2012). Although these attempts have yielded some progress in controlling seed dormancy and PHS, more work is required.

The objective of this study was to determine whether the $R H A 2 b$ gene from radish could improve the PHS tolerance of wheat. The RsRHA2b gene was used to improve seed dormancy and PHS tolerance in wheat.
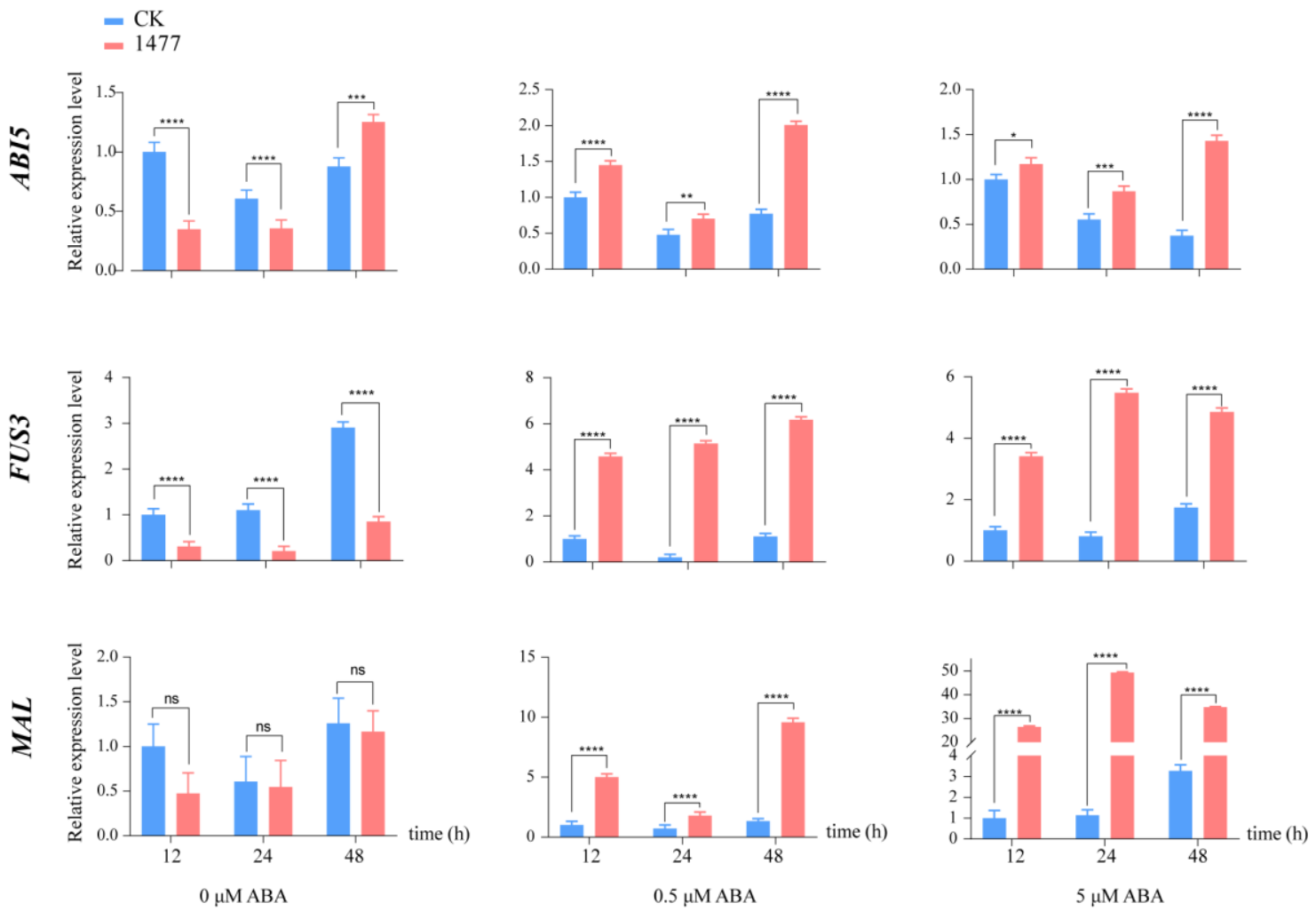

Figure 6. $q R T-P C R$ analysis of three genes in the T5 generation of transgenic line 1477 and nontransgenic Zheng 9023 is presented. RNA was isolated from the leaves at the second leaf stage of the plants. ABI5: a positive regulator of ABA signaling; MAL: involved in the pathways of $A B A$

signaling; FUS3: involved with the regulation of ABA biosynthesis. The symbol '*' indicates statistically differences $P<0.05$, the symbol '**' indicates statistically differences $P<0.01$; the symbol '***'indicates statistically differences $P<0.001$; the symbol '****' indicates statistically differences $P<0.0001$ (Two-way ANOVA, Sidak's multiple comparisons test) 
The success of the Agrobacterium-mediated transformation method used here provides a new technique for wheat genetic breeding (Zhao et al., 2006). In this study, the stem apex was used to improve Agrobacterium-mediated transformation. Basta was used to select the transgenic lines. The optimum half lethal concentration of basta had not been reported previously. Prior to this study, the concentration was analyzed using concentration gradient experiments. The phenotypes of plants treated with basta solutions of $80 \mathrm{mg} / \mathrm{L}, 100 \mathrm{mg} / \mathrm{L}, 120 \mathrm{mg} / \mathrm{L}, 140 \mathrm{mg} / \mathrm{L}, 160 \mathrm{mg} / \mathrm{L}, 180 \mathrm{mg} / \mathrm{L}, 200 \mathrm{mg} / \mathrm{L}$, $220 \mathrm{mg} / \mathrm{L}, 240 \mathrm{mg} / \mathrm{L}$, and $260 \mathrm{mg} / \mathrm{L}$ were recorded. The results showed that the optimum half lethal concentration of basta was $160 \mathrm{mg} / \mathrm{L}$. This method can provide genetically stable material for further application. As shown in Figure 3, the transgenic lines were rigorously screened using basta, PCR and RT-PCR. The seeds from pure transgenic lines, identified based on the segregation ratio of the integrated $R s R H A 2 b$ gene, were used for propagation.

Seed dormancy was stably inherited in transgenic wheat lines carrying the RHA2b gene from radish (Figs. 3 and 4). GIs and germination capacities were stably reduced in the T5 generation of transgenic wheat lines (Table 2). The transgenic lines in the present study had significantly increased tolerance to PHS, indicating that $R s R H A 2 b$ has an important role in regulating seed dormancy and PHS tolerance.

The resistance to dormancy and PHS is regulated by ABA anabolism and ABA signal transduction (Gubler et al., 2005). Additionally, studies on wheat mutants have shown that ABA sensitivity is directly associated with seed dormancy (Kawakami et al., 1997; Noda et al., 2002). Research on the physiological characteristics of wheat has shown that ABA synthesis is important for embryos to remain dormant. However, embryo dormancy is closely associated with the internal ABA content and the sensitivity of embryos to ABA (King, 1993; Morris et al., 1989; Walker-Simmons, 1987). Cultivars with strong dormancy have strong sensitivity to ABA (Morris et al., 1989; Walker-Simmons, 1987; Corbineau et al., 2000). These results demonstrate that both $\mathrm{ABA}$ signal transduction and $\mathrm{ABA}$ cascade effectors are important for maintaining dormancy and resisting PHS. Increased ABA sensitivity in transgenic wheat may increase survival rates.

In this study, the RsRHA2b-regulated ABA sensitivity apparently failed to impair the growth of transgenic wheat plants without exogenous ABA, but with exogenous ABA, smaller weight increases were observed (Table 3). Therefore, the exogenous RsRHA2b gene may regulate the ABA signaling pathway in transgenic wheat.

Although the AtRHA2b gene plays a positive role in regulating ABA signaling and stress responses, the regulatory factors and response regulators associated with $A t R H A 2 b$ are not known. Previous qRT-PCR assays have indicated that ABA treatment increased the expression of several ABA-inducible marker genes, including $R A B 18$, RD29A, KIN1, AtADH1, RD22 and RD29B in wild-type Arabidopsis (Li et al., 2011). The regulators associated with $\mathrm{ABA}$ and $\mathrm{ABA}$-inducible marker genes were chosen for analysis in transgenic wheat containing the RsRHA2b gene. In the present work, three genes, i.e., $A B I 5, F U S 3$ and $M A L$, were found to be significantly associated with $R H A 2 b$. ABI5, a bZIP transcription factor, is a positive regulator of ABA signaling, and its expression can be activated by exogenous ABA treatment (Suzuki et al., 2003; Kobayashi et al., 2008). FUS3 (FUSCA3) promotes dormancy and prevents the precocious germination of immature seeds by stimulating ABA synthesis (Chiu et al., 2012). MAL is involved in the metabolic pathways of ABA signaling (Huang et al., 2012). The expression of $A B I 5, F U S 3$ and $M A L$ significantly increased in the presence 
of $0.5 \mu \mathrm{mol} / \mathrm{L} \mathrm{ABA}$ and $5 \mu \mathrm{mol} / \mathrm{L} \mathrm{ABA}$ at the second leaf stage in transgenic wheat (Fig. 6). Thus, the RsRHA2b gene could positively regulate ABA signaling intermediates in transgenic wheat.

The grain germination rate of the over-expressed miR9678 wheat transgenic lines was significantly lower than that of the wild type, and the content of active gibberellin was reduced, leading to significantly increased resistance to spike germination (Guo et al., 2018). The miR9678 can mediate the splicing of long-segment non-coding RNA and produce a series of phasiRNA, which eventually leads to the change of gibberellin metabolism and its regulatory pathway by affecting the expression of gibberellin synthesis gene, thus controlling the germination rate of grain and spike germination (Guo et al., 2018). Based on transgenic wheat containing RsRHA2b, the reason for significantly increased tolerance to PHS can be explored by further work.

\section{Conclusion}

The RsRHA2b gene was integrated into the genomes of the wheat transformants. Transgenic wheat containing the RsRHA2b gene showed improved seed dormancy and PHS tolerance. RsRHA2b gene plays a significant role in regulating the genes associated with ABA signal transduction. This study provides a promising strategy for breeding wheat cultivars with improved seed dormancy and PHS tolerance using genetic engineering with the RsRHA $2 b$ gene. However, more work is needed to discover the mechanism of $R s R H A 2 b$-mediated PHS resistance.

Acknowledgements. This work was supported by the "Twelfth Five-Year" National Science and Technology Projects in Rural Areas (2013BADD04B01-02) and Henan Science and Technology Project (162102110007).

Conflict of interests. Authors state no conflict of interests.

\section{REFERENCES}

[1] Anderson, J. A., Sorrells, M. E., Tanksley, S. D. (1993): RFLP analysis of genomic regions associated with resistance to preharvest sprouting in wheat. - Crop Science 33: 453-459.

[2] Andreoli, C., Bassoi, M. C., Brunetta, D. (2006): Genetic control of seed dormancy and pre-harvest sprouting in wheat. - Scientia Agricola 63: 564-566.

[3] Bassoi, M. C., Flintham, J. (2005): Relationship between grain colour and preharvest sprouting-resistance in wheat. - Pesquisa Agropecuária Brasileira 40: 981-988.

[4] Borden, K. L. (2000): RING domains: master builders of molecular scaffolds? - Journal of Molecular Biology 295: 1103-1112.

[5] Bu, Q., Jiang, H., Li, C.-B., Zhai, Q., Zhang, J., Wu, X., Sun, J., Xie, Q., Li, C. (2008): Role of the Arabidopsis thaliana NAC transcription factors ANAC019 and ANAC055 in regulating jasmonic acid-signaled defense responses. - Cell Research 18: 756-767.

[6] Bu, Q., Li, H., Zhao, Q., Jiang, H., Zhai, Q., Zhang, J., Wu, X., Sun, J., Xie, Q., Wang, D., Li, C. (2009): The arabidopsis ring finger E3 ligase RHA2a is a novel positive regulator of abscisic acid signaling during seed germination and early seedling development. - Plant Physiology 150: 463-481.

[7] Castor, L., Frederiken, F. (1977): Seed moulding of grain sorghums caused by Fusarium and Curvularia. - Proc Annu Phytopathol Soc 4: 151. 
[8] Chen, C. X., Cai, S. B., Bai, G. H. (2008a): A major QTL controlling seed dormancy and pre-harvest sprouting resistance on chromosome $4 \mathrm{~A}$ in a Chinese wheat landrace. Molecular Breeding 21: 351-358.

[9] Chen, Q. J., Zhang, L. Q., Yang, Y. W., Yuan, Z. W., Xiang, Z. G., Zheng, Y. L., Peng, Z. S., Liu, D. C. (2008b): Dormancy spreads seed germination over a long period with a discontinuous procession in Aegilops tauschii, the D-genome donor species of bread wheat. - International Journal of Agricultural Research 3: 77-82.

[10] Chiu, R. S., Nahal, H., Provart, N. J., Gazzarrini, S. (2012): The role of the Arabidopsis FUSCA3 transcription factor during inhibition of seed germination at high temperature. BMC Plant Biology 12: 15.

[11] Corbineau, F., Benamar, A., Daniel, C. (2000): Changes in sensitivity to abscisic acid of the developing and maturing embryo of two wheat cultivars with different sprouting susceptibility. - Israel Journal of Plant Sciences 48: 189-197.

[12] Derera, N., Bhatt, G., Mcmaster, G. (1977): On the problem of pre-harvest sprouting of wheat. - Euphytica 26: 299-308.

[13] Dreher, K., Callis, J. (2007): Ubiquitin, hormones and biotic stress in plants. - Annals of Botany 99: 787-822.

[14] Gerjets, T., Scholefield, D., Foulkes, M. J., Lenton, J. R., Holdsworth, M. J. (2010): An analysis of dormancy, ABA responsiveness, after-ripening and pre-harvest sprouting in hexaploid wheat (Triticum aestivum, L.) caryopses. - Journal of Experimental Botany 61: 597-607.

[15] Grill, E., Himmelbach, A. (1998): ABA signal transduction. - Current Opinion in Plant Biology 1: 412-418.

[16] Groot, S. P., Karssen, C. M. (1992): Dormancy and germination of abscisic acid-deficient tomato seeds Studies with the Sitiens mutant. - Plant Physiology 99: 952-958.

[17] Gubler, F., Millar, A. A., Jacobsen, J. V. (2005): Dormancy release, ABA and pre-harvest sprouting. - Current Opinion in Plant Biology 8: 183-187.

[18] Guo, G., Liu, X., Sun, F., Cao, J., Huo, N., Wuda, B., Xin, M., Hu, Z., Du, J., Xia, R. (2018): Wheat miR9678 affects seed germination by generating phased siRNAs and modulating abscisic acid/gibberellin signaling. - The Plant Cell 30: 796-814.

[19] Holdsworth, M. J., Bentsink, L., Soppe, W. J. (2008): Molecular networks regulating Arabidopsis seed maturation, after-ripening, dormancy and germination. - New Phytologist 179: 33-54.

[20] Huang, T., Qu, B., Li, H. P., Zuo, D. Y., Zhao, Z. X., Liao, Y. C. (2012): A maize viviparous 1 gene increases seed dormancy and preharvest sprouting tolerance in transgenic wheat. - Journal of Cereal Science 55: 166-173.

[21] Hughes, K., Griffey, C., Parrish, D., Barbeau, W., Souza, E., Thomason, W. (2010): Preharvest sprouting tolerance in current soft red winter wheat cultivars. - Crop Science 50: 1449-1457.

[22] Jiang, H. L., Li, H. M., Bu, Q. Y., Li, C. Y. (2009): The RHA2a-interacting proteins ANAC019 and ANAC055 may play a dual role in regulating ABA response and jasmonate response. - Plant Signaling \& Behavior 4: 464-466.

[23] Kamthan, A., Chaudhuri, A., Kamthan, M., Datta, A. (2016): Genetically modified (GM) crops: milestones and new advances in crop improvement. - Theoretical \& Applied Genetics 129: 1639-1655.

[24] Kawakami, N., Miyake, Y., Noda, K. (1997): ABA insensitivity and low ABA levels during seed development of non-dormant wheat mutants. - Journal of Experimental Botany 48: 1415-1421.

[25] King, R., Von Wettstein-Knowles, P. (2000): Epicuticular waxes and regulation of ear wetting and pre-harvest sprouting in barley and wheat. - Euphytica 112: 157-166.

[26] King, R. W. (1993): Manipulation of grain dormancy in wheat. - Journal of Experimental Botany 44: 1059-1066. 
[27] Ko, J. H., Yang, S. H., Han, K. H. (2006): Upregulation of an Arabidopsis RING-H2 gene, XERICO, confers drought tolerance through increased abscisic acid biosynthesis. The Plant Journal 47: 343-355.

[28] Kobayashi, F., Maeta, E., Terashima, A., Takumi, S. (2008): Positive role of a wheat HvABI5 ortholog in abiotic stress response of seedlings. - Physiologia Plantarum 134: 74-86.

[29] Koornneef, M., Reuling, G., Karssen, C. (1984): The isolation and characterization of abscisic acid-insensitive mutants of Arabidopsis thaliana. - Physiologia Plantarum 61: 377-383.

[30] Kucera, B., Cohn, M. A., Leubner-Metzger, G. (2005): Plant hormone interactions during seed dormancy release and germination. - Seed Science Research 15: 281-307.

[31] Li, C. D., Ni, P. X., Francki, M., Hunter, A., Zhang, Y., Schibeci, D., Li, H., Tarr, A., Wang, J., Cakir, M. (2004): Genes controlling seed dormancy and pre-harvest sprouting in a rice-wheat-barley comparison. - Functional \& Integrative Genomics 4: 84-93.

[32] Li, D., Lyu, G., Lyu, J., Niu, H., Wang, X., Yin, J. (2019a): Cloning and characterization of a wheat RING finger gene TaRHA2b whose expression is up-regulated by ABA treatment. - Applied Ecology and Environmental Research 17: 7495-7510.

[33] Li, D. B., Zuo, N., Lyu, G. Z., Du, H. X., Niu, H. B., Yin, J. (2019b): Identification of characteristics of pre-harvest sprouting and differences of TaRHA2b gene sequence among different wheat varieties. - Journal of Henan Agricultural Sciences 48: 6-16.

[34] Li, H., Jiang, H., Bu, Q., Zhao, Q., Sun, J., Xie, Q., Li, C. (2011): The Arabidopsis RING finger E3 ligase RHA2b acts additively with RHA2a in regulating abscisic acid signaling and drought response. - Plant Physiology 156: 550-63.

[35] Li, Y. C., Ren, J. P., Cho, M. J., Zhou, S. M., Kim, Y. B., Guo, H. X., Wong, J. H., Niu, H. B., Kim, H. K., Morigasaki, S. (2009): The level of expression of thioredoxin is linked to fundamental properties and applications of wheat seeds. - Molecular Plant 2: 430-441.

[36] Lopez-Molina, L., Mongrand, S., Chua, N.-H. (2001): A postgermination developmental arrest checkpoint is mediated by abscisic acid and requires the ABI5 transcription factor in Arabidopsis. - Proceedings of the National Academy of Sciences 98: 4782-4787.

[37] Ma, L., Li, Z., Ren, T., Tang, Z., Yan, B., Ren, Z. (2014): Evaluation and validation of molecular marker associated with pre harvest sprouting tolerance in a RIL population. Journal of Triticeae Crops 34: 435-442.

[38] Ma, W., Zhang, C., Song, X., Feng, J., Cui, Z., Sun, D. (2016): Pre-harvest sprouting resistance in wheat from different wheat regions and its correlation with ear characteristics. - Journal of Triticeae Crops 36: 1269-1274.

[39] Major, B., Kettlewell, P., Scott, R. (2001): Mechanisms leading to excess alpha-amylase activity in wheat (Triticum aestivum, L) grain in the UK. - Journal of Cereal Science 33: 313-329.

[40] Mohan, A. (2008): Pre-harvest sprouting in cereals: a global scenario. - Current ScienceBangalore 94: 704.

[41] Moon, J., Parry, G., Estelle, M. (2004): The ubiquitin-proteasome pathway and plant development. - Plant Cell 16: 3181-3195.

[42] Morris, C., Moffatt, J., Sears, R., Paulsen, G. (1989): Seed dormancy and responses of caryopses, embryos, and calli to abscisic acid in wheat. - Plant physiology 90: 643-647.

[43] Munkvold, J. D., Tanaka, J., Benscher, D., Sorrells, M. E. (2009): Mapping quantitative trait loci for preharvest sprouting resistance in white wheat. - Theoretical and Applied Genetics 119: 1223-1235.

[44] Nambara, E., Marion-Poll, A. (2003): ABA action and interactions in seeds. - Trends in Plant Science 8: 213-217.

[45] Noda, K., Matsuura, T., Maekawa, M., Taketa, S. (2002): Chromosomes responsible for sensitivity of embryo to abscisic acid and dormancy in wheat. - Euphytica 123: 203-209.

[46] Ogbonnaya, F. C., Imtiaz, M., Ye, G., Hearnden, P. R., Hernandez, E., Eastwood, R. F., Van Ginkel, M., Shorter, S., Winchester, J. (2008): Genetic and QTL analyses of seed 
dormancy and preharvest sprouting resistance in the wheat germplasm CN10955. Theoretical and Applied Genetics 116: 891-902.

[47] Raghavendra, A. S., Gonugunta, V. K., Christmann, A., Grill, E. (2010): ABA perception and signalling. - Trends in Plant Science 15: 395-401.

[48] Saad, A. S., Li, X., Li, H. P., Huang, T., Gao, C. S., Guo, M. W., Cheng, W., Zhao, G. Y., Liao, Y. C. (2013): A rice stress-responsive NAC gene enhances tolerance of transgenic wheat to drought and salt stresses. - Plant Sci 203-204: 33-40.

[49] Schwechheimer, C., Schwager, K. (2004): Regulated proteolysis and plant development. - Plant Cell Reports 23: 353-364.

[50] Smalle, J., Vierstra, R. D. (2004): The ubiquitin 26S proteasome proteolytic pathway. Annu. Rev. Plant Biol. 55: 555-590.

[51] Somers, D., Ogbonnaya, F. C., Imtiaz, M., Depauw, R. M. (2007): Haplotype diversity of preharvest sprouting QTLs in wheat. - Genome 50: 107-118.

[52] Stone, S. L., Hauksdóttir, H., Troy, A., Herschleb, J., Kraft, E., Callis, J. (2005): Functional analysis of the RING-type ubiquitin ligase family of Arabidopsis. - Plant Physiology 137: 13-30.

[53] Suzuki, M., Ketterling, M. G., Li, Q.-B., Mccarty, D. R. (2003): Viviparous1 alters global gene expression patterns through regulation of abscisic acid signaling. - Plant Physiology 132: $1664-1677$.

[54] Tan, B. C., Schwartz, S. H., Zeevaart, J. A., Mccarty, D. R. (1997): Genetic control of abscisic acid biosynthesis in maize. - Proceedings of the National Academy of Sciences 94: 12235-12240.

[55] Torada, A., Amano, Y. (2002): Effect of seed coat color on seed dormancy in different environments. - Euphytica 126: 99-105.

[56] Vierstra, R. D. (2009): The ubiquitin-26S proteasome system at the nexus of plant biology. - Nature Reviews Molecular Cell Biology 10: 385-397.

[57] Walker-Simmons, M. (1987): ABA levels and sensitivity in developing wheat embryos of sprouting resistant and susceptible cultivars. - Plant physiology 84: 61-66.

[58] Xiao, S. H., Zhang, X. Y., Yan, C. S., Lin, H. (2002): Germplasm improvement for preharvest sprouting resistance in Chinese white-grained wheat: an overview of the current strategy. - Euphytica 126: 35-38.

[59] Yamaguchi Shinozaki, K., Shinozaki, K. (2006): Transcriptional regulatory networks in cellular responses and tolerance to dehydration and cold stresses. - Annu. Rev. Plant Biol. 57: 781-803.

[60] Zanetti, S., Winzeler, M., Keller, M., Keller, B., Messmer, M. (2000): Genetic analysis of pre-harvest sprouting resistance in a wheat $\times$ spelt cross. - Crop Science 40: 1406-1417.

[61] Zhang, X. R., Garreton, V., Chua, N.-H. (2005): The AIP2 E3 ligase acts as a novel negative regulator of ABA signaling by promoting $\mathrm{ABI} 3$ degradation. - Genes \& Development 19: 1532-1543.

[62] Zhang, Y., Yang, C., Li, Y., Zheng, N., Chen, H., Zhao, Q., Gao, T., Guo, H., Xie, Q. (2007): SDIR1 is a RING finger E3 ligase that positively regulates stress-responsive abscisic acid signaling in Arabidopsis. - The Plant Cell Online 19: 1912-1929.

[63] Zhao, T. J., Zhao, S. Y., Chen, H. M., Zhao, Q. Z., Hu, Z. M., Hou, B. K., Xia, G. M. (2006): Transgenic wheat progeny resistant to powdery mildew generated by Agrobacterium inoculum to the basal portion of wheat seedling. - Plant Cell Reports 25: 1199-1204.

[64] Zheng, Y., Chen, Z., Ma, L., Liao, C. (2018): The ubiquitin E3 ligase RHA2b promotes degradation of MYB30 in abscisic acid signaling. - Plant Physiology 178: 428-440.

[65] Zhu, J. K. (2002): Salt and drought stress signal transduction in plants. - Annual Review of Plant Biology 53: 247. 